\title{
The Characteristics and outcomes of patients with malignant pheochromocytoma and paraganglioma: Single-Center Experience
}

\author{
Asena Gökçay Canpolat ', [MD] \\ ORCID: 0000-0003-1186-2960 \\ Demet Çorapçıŏlu ', [MD] \\ ORCID: 0000-0003-0940-9147 \\ ${ }^{1}$ Ankara University, Faculty of Medicine, Department of
Endocrinology and Metabolism
}

Corresponding Author: Asena Gökçay Canpolat, MD. Ankara University School of Medicine, Department of Endocrinology and Metabolism, Ankara, Turkey.

E-mail: asena-gokcay@hotmail.com

Tel: 903125082108

Fax: 903123094505

\section{reO A B T RACT Cer}

Introduction: Metastatic pheochromocytoma (PCC) and paraganglioma (PGL) $[\mathrm{PPGL}]$ are rare neuroendocrine malignancies characterized by the presence of metastasis in nonchromaffin tissue. However, the data for clinical, histological, or biochemical predictors of metastatic behavior is scarce. So, we aimed to present the clinical features, treatment, and outcomes of our PPGL patients with metastatic behavior. Methods: A total of 38 patients with the diagnosis of PPGLs ( $n=35$ PCCs and $n=3$ PGLs) were enrolled in the study between 2010 and 2019. The demographics, clinical features, laboratory tests, radiological data, treatments, and outcomes of the patients were analyzed.

Results: The mean age was $38.3 \pm 15.3$ years, and the female to male ratio was $22 / 16$. Underlying etiology was hereditary in $60 \%$ of PCCs [multiple endocrine neoplasia (MEN) type 2A in 17 patients; MEN type 2B in 4 patients; and neurofibromatosis in one patient]. The metastatic disease was observed in 5 patients with PPGLs $(n=4$ of PCCs and $n=1$ of PGLs). The metastatic cases were sporadic all PPGL patients. The mean maximum size of the primary tumor of metastatic PPGL cases was $4.5 \pm 3.2 \mathrm{~cm}$. The sites of metastasis for PCCs were liver, bones, lungs, and lymph nodes in all cases. All metastatic patients underwent surgery. Cytotoxic chemotherapy and 131I- metaiodobenzylguanidine (MIBG) radionuclide therapy were given for all PCCs and external beam radiation for PGL patients. Three of the cases were stable with cytotoxic chemotherapy and 131I-MIBG, but one case exacerbated and died after a total of four cycles of $177 \mathrm{Lu}$ therapy.

Discussion: In our tertiary referral center, the majority of the patients who were followed with PPGLs have hereditary/genetic etiology. 10\% of our PPGL patients showed malignant features, which was consistent with the literature. Additionally, these patients should be managed in referral centers with a multidisciplinary approach providing chemotherapy and theranostic therapies.

Keywords: Pheochromocytoma, paraganglioma, metastatic disease, theranostic treatment

Received: 18 June 2020, Accepted: 23 June 2020

Published online: 30 June 2020

\section{INTRODUCTION}

Pheochromocytoma (PCC) and paraganglioma (PGL) [PPGLs] are rare neuroendocrine tumors of the autonomic nervous system [1 2]. The incidence of PCC is 2 to 8 per million persons per year, and the peak incidence occurs in the third to fifth decades of life [3 4]. Metastatic PPGL is characterized by the presence of metastatic disease in nonchromaffin tissue independent from being recurrent or locally invasive tumors [5]. Metastatic PPGLs have $a<1 / 1000$ 000 incidence, and the prevalence varies between 10 and $17 \%$ [6 7]. Approximately $10 \%$ of PCCs and up to $25 \%$ of thoracoabdominal and pelvic PGLs are 
metastatic [8]. The typical metastatic sites of PCCs are bones and lymph nodes, whereas hepatic metastases are the primary sites for PGLs [2].

The pathogenesis and progression of PPGLs are strongly influenced by the presence of specific genes [6]. The succinate dehydrogenase (SDH) gene B-related PGL has the highest metastatic potential (up to $50 \%$ ), whereas $3-5 \%$ of PGLs associated with MEN2 are metastatic [8]. Despite all these findings, there are no established clinical, histological, or biochemical predictors of metastatic behavior for PPGLs.

The treatment of metastatic PPGL is clinically meaningful because of its potential cardiovascular mortality and morbidity [7]. Management of PPGLs includes a combination of surgical resection, chemotherapy, external beam irradiation, thermal ablation, and radionuclide therapy with a multidisciplinary and systematic approach [5].

Thus, we aimed to present the clinical features, treatment, and outcomes of our PPGL patients with metastatic behavior.

\section{MATERIALS AND METHOD}

The patients diagnosed with either PCC or PGL (PPGL) between 2013 and 2019 were enrolled retrospectively. A total of 38 patients were included in the study. Subjects were classified as malignant according to the radiological evidence of metastasis. Biochemical tests, including 24-hour urinary metanephrines and catecholamines along with plasma catecholamines, were all recorded. 24-hour urinary catecholamine and metanephrine concentrations were assessed by Liquid Chromatography-Mass Spectrometry (LC-MS). Both computed tomography scan (CT), magnetic resonance imaging (MRI), and, if done, functional imaging [metaiodobenzylguanidine (MIBG) scan/scintigraphy] of the lesions were also evaluated. The results of the genetic analyses which could be performed in a subgroup of patients were also collected. The therapeutic options and their outcomes were recorded. Our institutional ethical committee approved the study.

Continuous variables were presented as mean \pm standard deviation, and categorical variables were presented as number (\%). All analyses were performed using the IBM SPSS software, version 25.0 (IBM Corp. Released 2015, Armonk, NY, USA). Since the urinary catecholamine levels were not normally distributed, nonparametric tests were conducted to compare these variables. The Mann-Whitney $\mathrm{U}$ test was used to compare urinary catecholamine levels between the metastatic and non-metastatic groups. A p-value of less than 0.05 was considered to show a statistically significant result.

\section{RESULTS}

A total of 38 cases with the diagnosis of PPGLs ( $n=35$ of PCCs and $n=3$ of PGLs) were enrolled in the study. The mean age was $38.3 \pm 15.3$ years, and the female to male ratio was $22 / 16$. Underlying etiology was hereditary in $60 \%$ of PCCs [multiple endocrine neoplasia (MEN) type 2A in 17 patients; MEN type 2B in 4 patients; and neurofibromatosis in one patient]. All of the PGL cases were sporadic. The mean age of cases with genetic $P C C$ s was $33.2 \pm 12.5$ years.

The involved location of the adrenal glands in PCC patients was at the left side for 9 patients, at the right side for 8 patients, and bilateral for 18 patients. A female predominance was observed in PCC cases $(F / M=19 / 16)$. A total of 7 patients were symptomatic before the diagnosis (PCC attack in six cases, and abdominal pain in one patient). The PCCs' sizes were ranged from $1 \mathrm{~cm}$ to $10 \mathrm{~cm}$ with a mean maximum size of $32 \pm 19.3 \mathrm{~cm}$ in magnetic resonance imaging (MRI). The mean computed tomography (CT) attenuation value was $44 \pm 13$ Hounsfield Units (HU) (unenhanced, precontrast) for PCCs. The urinary catecholamine and derivates for PPGLs were summarized in Table1. 
Table 1. The levels of urinary catecholamines and their metabolites in patients with PPGLs.

\begin{tabular}{|l|c|}
\hline Parameters & $2.5 \pm 1.18$ \\
\hline Urinary HVA $(0.5-6.2 \mathrm{mg} / 24 \mathrm{~h})$ & $281(90-10486)$ \\
\hline Urinary normetanephrines $(88-444 \mu \mathrm{g} / \mathrm{d})$ & $95(14.4-310)$ \\
\hline Urinary metanephrines $(52-341 \mu \mathrm{g} / \mathrm{d})$ & $4.1(1.6-57.5)$ \\
\hline Urinary VMA $(1.8-6.7 \mathrm{mg} / 24 \mathrm{~h})$ & $11(2.4-24)$ \\
\hline Urinary epinephrine $(2-22 \mathrm{mg} / \mathrm{d})$ & $50(16-446)$ \\
\hline Urinary norepinephrine $(20-81 \mu \mathrm{g} / \mathrm{d})$ & \\
\hline
\end{tabular}

HVA: Homovanillic acid VMA: Vanillylmandelic acid H: hour

\section{DISQUSSION}

The median maximum size of the PGLs was 2 (2-2.5) $\mathrm{cm}$, and all the patients were female. PGLs were located at the level of the neck in all cases. Only one patient was symptomatic among PGLs.

The metastatic disease was seen in a total of 5 patients ( $n=4$ of PCCs and $n=1$ of PGLs). The metastatic cases were sporadic both in PCC and PGL patients. The mean maximum size of the primary tumor of metastatic PPGL cases was $4.5 \pm 3.2 \mathrm{~cm}$. The sites of metastasis for PCCs were liver, bones, lungs, and lymph nodes in all cases. One patient with PGL had metastasis at lymph nodes of the neck and skull.

The mean duration of the follow-up of metastatic PPGLs was $8.8 \pm 7$ years. The treatment of metastatic disease was radiation therapy for the patient with PGL and 131I-MIBG, cytotoxic chemotherapy (cisplatin and etoposide), and peptide receptor radionuclide therapy (177Lu-DOTATATE) for PCCs. Surgical resection was performed for primary tumor in all metastatic cases. All of the PCCs received conventional cytotoxic chemotherapy at the beginning of the metastatic disease and then received 131I-MIBG radionuclide therapy. Three of the cases were stable with cytotoxic chemotherapy and 131-I-MIBG, but one case deceased under I131MIBG targeted therapy. After a total of $800 \mathrm{Mci}$, four cycles of 177LuDOTATATE was planned for this patient. The dimension of the metastasis was stable, but she was symptomatic with high levels of catecholamines. Soon after she received two cycles of 177Lu-DOTATATE, she died because of uncontrolled hypertension and intracranial hemorrhage during a PCC attack. She died after ten years of her initial diagnosis.
Metastatic PPGLs can be diagnosed by radiological evaluation on the long term follow-up of the patients. Although there are no established clinical, histological, or biochemical predictors of metastatic behavior, some of the authors claimed that the presence of some clinical predictors could guide clinicians to assess for metastasis. PCCs larger than 5 $\mathrm{cm}$, extra-adrenal location of the primary PCC, PGLs at any size, and mutation of SDHB are some risk factors identified for metastasis [9]. In our study, metastatic PPGLs did not present any of the risk factors mentioned above, except one case presented with a $10 \mathrm{~cm}$ diameter of PCC. They were all sporadic cases with relatively smaller dimensions of tumors mentioned above and without genetic predilection. Germ-line or somatic mutations of the SDHB gene are most relevant with metastatic PGGLS with a percentage of $30-44 \%$ [10]. However, we could not determine such a causal relationship in our patients. Also, the pathological evaluation did not reveal any prognostic indicators for these patients. It is a fact that pathological differentiation and proliferative index both have no prognostic value and guidance for treatment type in metastatic PPGLs [9].

The liver $(n=1)$, bones $(n=1)$, lungs $(n=1)$, and lymph nodes $(n=4)$ were the metastatic sites in our patients. Lymph node involvement was the most common site for metastasis, similar to the literature. Metastasis sites in PGGLs are lymph nodes (80\%), bones $(71 \%)$, liver (50\%), and lungs $(50 \%)$, respectively [11].

The mean follow-up of metastatic disease was $106 \pm 84$ months. All patients except a metastatic 
PCC case are still alive. A meta-analysis reported a five-year mortality rate of $37 \%$ and a 10 -year mortality rate of $29 \%$ [8]. In contrast, a novel and a large cohort of metastatic PGGLs revealed a disease-specific 5 -year survival rate of $88.2 \%$ and a 10 -year disease-specific survival rate of $77.9 \%$, indicating an excellent prognosis despite having malignant PPGLs [2]. Our findings were also consistent with these findings regarding survival. However, our study sample size was too small for comparison. As a general consideration, watchful waiting may be suitable for asymptomatic patients without disease progression. Among various treatment options, each one can be applied as a single or combination therapy in progressive metastatic disease [8]. First of all, control of hormone and tumor-related symptoms are the mainstay of the therapy. As cardiovascular complications and even death can occur due to excess catecholamine release, administration of $a$ adrenergic receptor blockers soon after $\beta$ adrenergic receptor blockers can provide control in catecholamine-related symptoms [8]. Other targets of the therapy are the reduction of biochemicals, and control of tumor growth.

Owing to the rarity of the disease and lack of prospectively designed studies, management of the metastatic PPGLs mostly depend on retrospective study results. Surgical resection is a curative treatment for PPGLs, and resection can ensure improvement in the survival response to systemic therapies and the achievement of biochemical control. An aggressive resection for PPGLs with the intra-abdominal disease is found to be more likely to achieve remission and biochemical response [12]. In our study, surgical debulking was performed for all of the PPGLs, and they had improvement both in symptoms and biochemical parameters. External beam radiation therapy (EBRT) is another treatment choice for stabilization of local tumor growth in nonresponder PGGLs for current systemic therapies [13]. EBRT was applied for the metastatic PGL in our study, who did not respond well to resection and chemotherapy.

MIBG with both 1131 or I123 is in use for functional imaging of PGGLs. While I131 MIBG has a sensitivity of $77 \%-90 \%$ and specificity of $95 \%-100 \%$, 1123 MIBG has a sensitivity of $83 \% 100 \%$ and specificity of 95\%-100\% for detecting PCC [14]. So, it can be used to determine the patients who are a candidate for radionuclide therapy with I131MIBG. A meta-analysis reported a $30 \%$ of patients treated with I131MIBG radionuclide therapy had improvement for symptoms and disease stabilization, and $35-67 \%$ had a biochemical response [15]. Another peptide-receptor radionuclide therapy is 177Lu -DOTATATE. The most extensive study of this treatment reported $78 \%$ of patients with stable disease and $7 \%$ of patients with partial response. All of our PCC patients received radionuclide therapy with I131MIBG, and stable three $(75 \%)$ of them were stable. However, one case worsened with increment both in size and number of metastasis. This case only received $177 \mathrm{Lu}$ -DOTATATE for four cycles, and she declined during the therapy until her death.

In cases of unresectable and progressive disease, systemic chemotherapy is indicated. The most common regimen for metastatic PGGLs is cyclophosphamide-vincristine-dacarbazine (CVD). The response rates between 25\%-55\% were reported for CVD regimen [9]. The side effects and toxicities are factors limiting their usage; temozolomide is proposed for a maintenance regimen. All of our patients received chemotherapy at the beginning of the therapy. The patients did not well tolerate the therapy, and it was switched to radionuclide therapy with 131MIBG. None of our patients received temozolomide. Tyrosine kinase inhibitors, interferon a, immunotherapy are under investigation for metastatic PGGLs.

The main limitations of our study were the small sample size of the metastatic group and the paucity of patients with being sporadic. Nevertheless, it was the first and the largest study evaluating the characteristics and treatment modalities of metastatic PGGLs in our country.

There is still inconclusive data about the biological behavior and risk factors for metastatic disease. Although disease-specific and overall survival rates were reported to be low until now, recently, we can see better survival rates in long and comprehensive 
studies alike, our study [2].

The management of metastatic PPGLs should be individualized because of differences in the course of the disease, sites of the metastasis, and risk factors for prognosis and treatment should be administered in the case-based decision. Also, these patients should be evaluated and treated by a multidisciplinary team.

\section{CONFUCT OF INTEREST STATEMENT}

The authors declare that there is no conflict of interest.
[1] Koopman K, Gaal J, de Krijger RR. Pheochromocytomas and Paragangliomas: New Developments with Regard to Classification, Genetics, and Cell of Origin. Cancers 2019;11(8): 1070.

[2] Hamidi O, Young WF, Jr., Iniguez-Ariza NM, et al. Malignant Pheochromocytoma and Paraganglioma: 272 Patients Over 55 Years. J Clin Endocrinol Metab 2017;102(9):3296-305.

[3] Beard CM, Sheps SG, Kurland LT, et al. Occurrence of pheochromocytoma in Rochester, Minnesota, 1950 through 1979. Mayo Clin Proc 1983;58(12):802-4

[4] Neumann HP, Bausch B, McWhinney SR, et al. Germ-line mutations in nonsyndromic pheochromocytoma. $\mathrm{N}$ Engl J Med 2002;346(19):1459-66.

[5] Neumann HPH, Young WF, Jr., Eng C. Pheochromocytoma and Paraganglioma. N Engl J Med 2019;381(6):552-65.

[6] Angelousi A, Kassi E, Zografos G, et al. Metastatic pheochromocytoma and paraganglioma. Eur J Clin Invest 2015;45(9):986-97.

[7] LendersJW,DuhQY,EisenhoferG,etal.Pheochromocytoma and paraganglioma: an endocrine society clinical practice guideline. J Clin Endocrinol Metab 2014;99(6):1915-42.

[8] Hamidi O. Metastatic pheochromocytoma and paraganglioma: recent advances in prognosis and management. Curr Opin Endocrinol Diabetes Obes 2019;26(3):146-54.
[9] Baudin E, Habra MA, Deschamps F, et al. Therapy of endocrine disease: treatment of malignant pheochromocytoma and paraganglioma. Eur J Endocrinol 2014;171(3):R111-22.

[10] Plouin PF, Fitzgerald P, Rich T, et al. Metastatic pheochromocytoma and paraganglioma: focus on therapeutics. Horm Metab Res 2012;44(5):390-9.

[11] jimenez C, Rohren E, Habra MA, et al. Current and future treatments for malignant pheochromocytoma and sympathetic paraganglioma. Curr Oncol Rep 2013;15(4):356-71.

[12] Ellis RJ, Patel D, Prodanov T, et al. Response after surgical resection of metastatic pheochromocytoma and paraganglioma: can postoperative biochemical remission be predicted? J Am Coll Surg 2013;217(3):489-96.

[13] Vogel J, Atanacio AS, Prodanov T, et al. External beam radiation therapy in treatment of malignant pheochromocytoma and paraganglioma. Front Oncol 2014;4:166.

[14] Ilias I, Divgi C, Pacak K. Current role of metaiodobenzylguanidine in the diagnosis of pheochromocytoma and medullary thyroid cancer. Semin Nucl Med 2011;41(5):364-8.

[15] [Jasim S, Jimenez C. Metastatic pheochromocytoma and paraganglioma: Management of endocrine manifestations, surgery and ablative procedures, and systemic therapies. Best Pract Res Clin Endocrinol Metab 2019:101354. 